Kragujevac Journal of Mathematics

Volume 45(2) (2021), PAges 191-202.

\title{
SOME REFINEMENTS OF THE NUMERICAL RADIUS INEQUALITIES VIA YOUNG INEQUALITY
}

\author{
Z. HEYDARBEYGI ${ }^{1}$ AND M. AMYARI ${ }^{1 *}$
}

\begin{abstract}
In this paper, we get an improvement of the Hölder-McCarthy operator inequality in the case when $r \geq 1$ and refine generalized inequalities involving powers of the numerical radius for sums and products of Hilbert space operators.
\end{abstract}

\section{INTRODUCTION}

Let $(\mathcal{H},\langle\cdot, \cdot\rangle)$ be a complex Hilbert space and $B(\mathcal{H})$ denote the $C^{*}$-algebra of all bounded linear operators on $\mathcal{H}$. Recall that for $A \in B(\mathcal{H}), W(A)=\{\langle A x, x\rangle$ : $x \in \mathcal{H},\|x\|=1\}, w(A)=\sup \{|\lambda|: \lambda \in W(A)\}$ and $\|A\|=\sup \{\|A x\|:\|x\|=1\}$, denote the numerical range, the numerical radius and the usual operator norm of $A$, respectively. Also an operator $A \in B(\mathcal{H})$ is said to be positive if $\langle A x, x\rangle \geqslant 0$ for each $x \in \mathcal{H}$ and, in this case, is denoted by $A \geqslant 0$.

It is well-known that $\overline{W(A)}$ is a convex subset of the complex plane that contains the convex hull spectrum of $A$ (see $[4$, p. 7]). It is known that $w(\cdot)$ defines a norm on $B(\mathcal{H})$, which is equivalent to the usual operator norm $\|\cdot\|[4$, Theorem 1.3-1]. For $A \in B(\mathcal{H})$, we have

$$
\frac{1}{2}\|A\| \leq w(A) \leq\|A\| .
$$

The inequalities in (1.1) have been improved by many mathematicians, (see [2, 7, 10, $13,17-19])$.

Key words and phrases. Bounded linear operator, Hilbert space, norm inequality, numerical radius inequality.

2010 Mathematics Subject Classification. Primary: 47A12, 47A30. Secondary: 47A63.

DOI 10.46793/KgJMat2102.191H

Received: July 27, 2018.

Accepted: November 06, 2018. 
Kittaneh in $[7,8]$ showed that if $A \in B(\mathcal{H})$, then

$$
w(A) \leq \frac{1}{2}\left\||A|+\left|A^{*}\right|\right\| \leq \frac{1}{2}\left(\|A\|+\left\|A^{2}\right\|^{\frac{1}{2}}\right),
$$

where $|A|^{2}=A^{*} A$, and

$$
\frac{1}{4}\left\|A^{*} A+A A^{*}\right\| \leq w^{2}(A) \leq \frac{1}{2}\left\|A^{*} A+A A^{*}\right\| .
$$

He also obtained the following generalizations of the first inequality in (1.2) and the second inequality in (1.3):

$$
w^{r}(A) \leq \frac{1}{2}\left\||A|^{2 \lambda r}+\left|A^{*}\right|^{2(1-\lambda) r}\right\|
$$

and

$$
w^{2 r}(A) \leq\left\|\lambda|A|^{2 r}+(1-\lambda)\left|A^{*}\right|^{2 r}\right\|,
$$

where $0<\lambda<1$, and $r \geq 1$ in [9, Theorem 1, Theorem 2], respectively.

In Section 2 of this paper, we get an improvement of the Hölder-McCarthy operator inequality in the case when $r \geq 1$ and refine inequality (1.4) for $r \geq 1$ and inequality (1.5) for $r \geq 2$, see $([3,12,16])$. In addition, we establish some improvements of norm and numerical radius inequalities for sums and powers of operators acting on a Hilbert space in Section 3. For recent work on the numerical radius inequalities, we refer the reader to $[13-15,18]$.

\section{Refinements of the HÖlder-McCarthy Operator Inequality}

In this section, we obtain an improvement of Hölder-McCarthy's operator inequality in the case when $r \geq 1$ and get some improvements of numerical radius inequalities for Hilbert space operators. The following lemmas are essential for our investigation. The first lemma is a simple consequence of the Jensen inequality for convex function $f(t)=t^{r}$, where $r \geq 1$.

Lemma 2.1. ([13, Lemma 2.1]). Let $a, b \geq 0$ and $0 \leq \lambda \leq 1$. Then

$$
a^{\lambda} b^{1-\lambda} \leq \lambda a+(1-\lambda) b \leq\left(\lambda a^{r}+(1-\lambda) b^{r}\right)^{\frac{1}{r}}, \quad \text { for } r \geq 1 .
$$

The second lemma is known as a generalized mixed Schwarz inequality.

Lemma 2.2. ([8, Lemma 5]). Let $A \in B(\mathcal{H})$ and $x, y \in \mathcal{H}$ be two vectors and $0 \leq \lambda \leq 1$. Then

$$
|\langle A x, y\rangle|^{2} \leq\left\langle|A|^{2 \lambda} x, x\right\rangle\left\langle\left|A^{*}\right|^{2(1-\lambda)} y, y\right\rangle .
$$

The third lemma follows from the spectral theorem for positive operators and the Jensen inequality and is known as the Hölder McCarthy inequality.

Lemma 2.3. ([13, Lemma 2.2]). Suppose that $A$ is a positive operator in $B(\mathcal{H})$ and $x \in \mathcal{H}$ is any unit vector. Then

(i) $\langle A x, x\rangle^{r} \leq\left\langle A^{r} x, x\right\rangle$ for $r \geq 1$; 
(ii) $\left\langle A^{r} x, x\right\rangle \leq\langle A x, x\rangle^{r}$ for $0<r \leq 1$.

The last lemma is an improvement of Hölder-McCarthy's inequality.

Lemma 2.4. ([6, Corollary 3.1]). Let $A$ be a positive operator on $\mathcal{H}$. If $x \in \mathcal{H}$ is a unit vector, then

$$
\langle A x, x\rangle^{r} \leq\left\langle A^{r} x, x\right\rangle-\left\langle|A-\langle A x, x\rangle|^{r} x, x\right\rangle, \quad \text { for } r \geq 2 .
$$

The next theorem is a refinement of inequality (1.5) for $r \geq 2$.

Theorem 2.1. If $A \in B(\mathcal{H}), 0<\lambda<1$ and $r \geq 2$, then

$$
w^{2 r}(A) \leq\left\|\lambda|A|^{2 r}+(1-\lambda)\left|A^{*}\right|^{2 r}\right\|-\inf _{\|x\|=1} \zeta(x),
$$

where

$$
\zeta(x)=\left\langle\left(\left.\lambda|| A\right|^{2}-\left.\left\langle|A|^{2} x, x\right\rangle\right|^{r}+\left.(1-\lambda)|| A^{*}\right|^{2}-\left.\left\langle\left|A^{*}\right|^{2} x, x\right\rangle\right|^{r}\right) x, x\right\rangle .
$$

Proof. Let $x \in \mathcal{H}$ be a unit vector.

$$
\begin{aligned}
|\langle A x, x\rangle|^{2} \leq & \left\langle|A|^{2 \lambda} x, x\right\rangle\left\langle\left|A^{*}\right|^{2(1-\lambda)} x, x\right\rangle \quad \text { (by Lemma 2.2) } \\
\leq & \left\langle|A|^{2} x, x\right\rangle^{\lambda}\left\langle\left|A^{*}\right|^{2} x, x\right\rangle^{1-\lambda} \quad(\text { by Lemma 2.3 (ii) }) \\
\leq & \left(\lambda\left\langle|A|^{2} x, x\right\rangle^{r}+(1-\lambda)\left\langle\left|A^{*}\right|^{2} x, x\right\rangle^{r}\right)^{\frac{1}{r}} \quad(\text { by Lemma 2.1) } \\
\leq & \left(\lambda\left(\left\langle|A|^{2 r} x, x\right\rangle-\left\langle\left.|| A\right|^{2}-\left.\left\langle|A|^{2} x, x\right\rangle\right|^{r} x, x\right\rangle\right)\right. \\
& \left.+(1-\lambda)\left(\left\langle\left|A^{*}\right|^{2 r} x, x\right\rangle-\left\langle\left.|| A^{*}\right|^{2}-\left.\left\langle\left|A^{*}\right|^{2} x, x\right\rangle\right|^{r} x, x\right\rangle\right)\right)^{\frac{1}{r}}
\end{aligned}
$$

(by Lemma 2.4).

Hence,

$$
\begin{aligned}
|\langle A x, x\rangle|^{2 r} \leq & \lambda\left(\left\langle|A|^{2 r} x, x\right\rangle-\left\langle\left.|| A\right|^{2}-\left.\left\langle|A|^{2} x, x\right\rangle\right|^{r} x, x\right\rangle\right) \\
& +(1-\lambda)\left(\left\langle\left|A^{*}\right|^{2 r} x, x\right\rangle-\left\langle\left.|| A^{*}\right|^{2}-\left.\left\langle\left|A^{*}\right|^{2} x, x\right\rangle\right|^{r} x, x\right\rangle\right) .
\end{aligned}
$$

By taking supremum over $x \in \mathcal{H}$ with $\|x\|=1$, we get the desired relation.

Recall that the Young inequality says that if $a, b \geq 0$ and $\lambda \in[0,1]$, then

$$
(1-\lambda) a+\lambda b \geq a^{1-\lambda} b^{\lambda} .
$$

Many mathematicians improved the Young inequality and its reverse. Kober [11], proved that for $a, b>0$

$$
(1-\lambda) a+\lambda b \leq a^{1-\lambda} b^{\lambda}+(1-\lambda)(\sqrt{a}-\sqrt{b})^{2}, \quad \lambda \geq 1
$$

By using (2.1), we obtain a refinement of the Hölder-McCarthy inequality. 
Lemma 2.5. Let $A \in B(\mathcal{H})$ be a positive operator. Then

$$
\langle A x, x\rangle^{\lambda}\left(1+2(\lambda-1)\left(1-\frac{\left\langle A^{\frac{1}{2}} x, x\right\rangle}{\langle A x, x\rangle^{\frac{1}{2}}}\right)\right) \leq\left\langle A^{\lambda} x, x\right\rangle
$$

for any $\lambda \geq 1$ and $x \in \mathcal{H}$ with $\|x\|=1$.

Proof. Applying functional calculus for the positive operator $A$ in (2.1), we get

$$
(1-\lambda) a I+\lambda A \leq a^{1-\lambda} A^{\lambda}+(1-\lambda)\left(a I+A-2 \sqrt{a} A^{\frac{1}{2}}\right) .
$$

The above inequality is equivalent to

$$
(1-\lambda) a+\lambda\langle A x, x\rangle \leq a^{1-\lambda}\left\langle A^{\lambda} x, x\right\rangle+(1-\lambda)\left(a+\langle A x, x\rangle-2 \sqrt{a}\left\langle A^{\frac{1}{2}} x, x\right\rangle\right),
$$

for any $x \in \mathcal{H}$ with $\|x\|=1$. By substituting $a=\langle A x, x\rangle$ in (2.3), we get

$$
\langle A x, x\rangle \leq\langle A x, x\rangle^{1-\lambda}\left\langle A^{\lambda} x, x\right\rangle+2(1-\lambda)\langle A x, x\rangle\left(1-\frac{\left\langle A^{\frac{1}{2}} x, x\right\rangle}{\langle A x, x\rangle^{\frac{1}{2}}}\right) .
$$

By rearranging terms, we get the desired result (2.2).

Note that by the Hölder-McCarthy inequality, $1 \geq 1-\frac{\left\langle A^{\frac{1}{2}} x, x\right\rangle}{\langle A x, x\rangle^{\frac{1}{2}}} \geq 0$. Hence, the following chain of inequalities are true:

$$
\langle A x, x\rangle^{\lambda} \leq\langle A x, x\rangle^{\lambda}\left(1+2(\lambda-1)\left(1-\frac{\left\langle A^{\frac{1}{2}} x, x\right\rangle}{\langle A x, x\rangle^{\frac{1}{2}}}\right)\right) \leq\left\langle A^{\lambda} x, x\right\rangle,
$$

where $A$ is positive and $\lambda \geq 1$. One can easily see that

$$
1-\frac{\left\langle A^{\frac{1}{2}} x, x\right\rangle}{\langle A x, x\rangle^{\frac{1}{2}}} \geq \inf \left\{1-\frac{\left\langle A^{\frac{1}{2}} x, x\right\rangle}{\langle A x, x\rangle^{\frac{1}{2}}}: x \in \mathcal{H},\|x\|=1\right\} \text {. }
$$

So,

$$
1+2(\lambda-1)\left(1-\frac{\left\langle A^{\frac{1}{2}} x, x\right\rangle}{\langle A x, x\rangle^{\frac{1}{2}}}\right) \geq 1+2(\lambda-1) \text { inf }\left\{1-\frac{\left\langle A^{\frac{1}{2}} x, x\right\rangle}{\langle A x, x\rangle^{\frac{1}{2}}}: x \in \mathcal{H},\|x\|=1\right\} .
$$

If we denote the right-hand side of inequality $(2.4)$ by $\zeta(x)$, then from inequality (2.2), we get

$$
\langle A x, x\rangle^{\lambda} \leq \frac{1}{\zeta}\left\langle A^{\lambda} x, x\right\rangle, \quad \lambda \geq 1
$$

The following theorem is an improvement of inequality (1.4).

Theorem 2.2. Let $A \in B(\mathcal{H})$ be an invertible operator, $0<\lambda<1$ and $r>1$. If for each unit vector $x \in \mathcal{H}$

$$
\zeta(x)=\left(1+2(r-1)\left(1-\frac{\left\langle|A|^{\lambda} x, x\right\rangle}{\left\langle|A|^{2 \lambda} x, x\right\rangle^{\frac{1}{2}}}\right)\right)
$$


and

then

$$
\gamma(x)=\left(1+2(r-1)\left(1-\frac{\left\langle\left|A^{*}\right|^{(1-\lambda)} x, x\right\rangle}{\left\langle\left|A^{*}\right|^{2(1-\lambda)} x, x\right\rangle^{\frac{1}{2}}}\right)\right),
$$

$$
w^{r}(A) \leq \frac{1}{2 \mu}\left\||A|^{2 \lambda r}+\left|A^{*}\right|^{2(1-\lambda) r}\right\|
$$

where $\zeta=\inf _{\|x\|=1} \zeta(x), \gamma=\inf _{\|x\|=1} \gamma(x)$ and $\mu=\min \{\zeta, \gamma\}$.

Proof. Let $x \in \mathcal{H}$ be a unit vector. Then

$$
\begin{aligned}
|\langle A x, x\rangle| & \leq\left\langle|A|^{2 \lambda} x, x\right\rangle^{\frac{1}{2}}\left\langle\left|A^{*}\right|^{2(1-\lambda)} x, x\right\rangle^{\frac{1}{2}} \\
& \leq\left(\frac{\left\langle|A|^{2 \lambda} x, x\right\rangle^{r}+\left\langle\left|A^{*}\right|^{2(1-\lambda)} x, x\right\rangle^{r}}{2}\right)^{\frac{1}{r}} \\
& \leq\left(\frac{1}{2}\left(\frac{1}{\zeta}\left\langle|A|^{2 r \lambda} x, x\right\rangle+\frac{1}{\gamma}\left\langle\left|A^{*}\right|^{2 r(1-\lambda)} x, x\right\rangle^{r}\right)\right)^{\frac{1}{r}} .
\end{aligned}
$$

Hence,

$$
|\langle A x, x\rangle|^{r} \leq \frac{1}{2 \mu}\left\langle\left(|A|^{2 \lambda r}+\left|A^{*}\right|^{2(1-\lambda) r}\right) x, x\right\rangle .
$$

By taking supremum over $x \in \mathcal{H}$ with $\|x\|=1$, we get the desired relation.

\section{Inequalities for Sums and Products of Operators}

In this section, we recall that some general result for the product of operators from $[5]$.

If $A, B \in B(\mathcal{H})$, then

$$
w(A B) \leq 4 w(A) w(B) .
$$

If $A$ is an isometry and $A B=B A$, or a unitary operator that commutes with another operator $B$, then

$$
w(A B) \leq w(B),
$$

(see [4, Corollary 2.5-3]). Dragomir in [1, Theorem 2] showed that for $A, B \in B(\mathcal{H})$, any $\lambda \in(0,1)$ and $r \geq 1$

$$
|\langle A x, B y\rangle|^{2 r} \leq \lambda\left\langle\left(A^{*} A\right)^{\frac{r}{\lambda}} x, x\right\rangle+(1-\lambda)\left\langle\left(B^{*} B\right)^{\frac{r}{1-\lambda}} y, y\right\rangle,
$$

where $x, y \in \mathcal{H}$, with $\|x\|=\|y\|=1$.

Let $A, B \in B(\mathcal{H})$. The Schwarz inequality states that

$$
|\langle A x, B y\rangle|^{2} \leq\langle A x, A x\rangle\langle B y, B y\rangle, \quad \text { for all } x, y \in \mathcal{H} .
$$

We get the following refinements of inequality (3.1) for $r \geq 2$. 
Lemma 3.1. For $A, B \in B(\mathcal{H}), 0<\lambda<1$ and $r \geq 2$

$$
\begin{aligned}
|\langle A x, B y\rangle|^{2 r} \leq & \lambda\left\langle\left(A^{*} A\right)^{\frac{r}{\lambda}} x, x\right\rangle-\lambda\left\langle\left|\left(A^{*} A\right)^{\frac{1}{\lambda}}-\left\langle\left(A^{*} A\right)^{\frac{1}{\lambda}} x, x\right\rangle\right|^{r} x, x\right\rangle+(1-\lambda) \\
& \times\left\langle\left(B^{*} B\right)^{\frac{r}{1-\lambda}} y, y\right\rangle-(1-\lambda)\left\langle\left|\left(B^{*} B\right)^{\frac{1}{1-\lambda}}-\left\langle\left(B^{*} B\right)^{\frac{1}{1-\lambda}} y, y\right\rangle\right|^{r} y, y\right\rangle,
\end{aligned}
$$

for any $x, y \in \mathcal{H}$, with $\|x\|=\|y\|=1$.

Proof. For any unit vectors $x, y \in \mathcal{H}$, we have $\left|\left\langle\left(B^{*} A\right) x, y\right\rangle\right|^{2} \leq\left\langle\left(A^{*} A\right) x, x\right\rangle\left\langle\left(B^{*} B\right) y, y\right\rangle \quad$ (by Schwarz inequality)

$$
\begin{aligned}
= & \left\langle\left(\left(A^{*} A\right)^{\frac{1}{\lambda}}\right)^{\lambda} x, x\right\rangle\left\langle\left(\left(B^{*} B\right)^{\frac{1}{1-\lambda}}\right)^{1-\lambda} y, y\right\rangle \\
\leq & \left\langle\left(A^{*} A\right)^{\frac{1}{\lambda}} x, x\right\rangle^{\lambda}\left\langle\left(B^{*} B\right)^{\frac{1}{1-\lambda}} y, y\right\rangle^{1-\lambda} \quad(\text { by Lemma 2.3) } \\
\leq & \left(\lambda\left\langle\left(A^{*} A\right)^{\frac{1}{\lambda}} x, x\right\rangle^{r}+(1-\lambda)\left\langle\left(B^{*} B\right)^{\frac{1}{1-\lambda}} y, y\right\rangle^{r}\right)^{\frac{1}{r}} \quad(\text { by Lemma 2.1) } \\
\leq & \left(\lambda\left\langle\left(A^{*} A\right)^{\frac{r}{\lambda}} x, x\right\rangle-\lambda\left\langle\left|\left(A^{*} A\right)^{\frac{1}{\lambda}}-\left\langle\left(A^{*} A\right)^{\frac{1}{\lambda}} x, x\right\rangle\right|^{r} x, x\right\rangle\right. \\
& +(1-\lambda)\left\langle\left(B^{*} B\right)^{\frac{r}{1-\lambda}} y, y\right\rangle \\
& \left.-(1-\lambda)\left\langle\left|\left(B^{*} B\right)^{\frac{1}{1-\lambda}}-\left\langle\left(B^{*} B\right)^{\frac{1}{1-\lambda}} y, y\right\rangle\right|^{r} y, y\right\rangle\right)^{\frac{1}{r}} \quad \text { (by Lemma 2.4). }
\end{aligned}
$$

Therefore,

$$
\begin{aligned}
|\langle A x, B y\rangle|^{2 r} \leq & \lambda\left\langle\left(A^{*} A\right)^{\frac{r}{\lambda}} x, x\right\rangle-\lambda\left\langle\left|\left(A^{*} A\right)^{\frac{1}{\lambda}}-\left\langle\left(A^{*} A\right)^{\frac{1}{\lambda}} x, x\right\rangle\right|^{r} x, x\right\rangle \\
& +(1-\lambda)\left\langle\left(B^{*} B\right)^{\frac{r}{1-\lambda}} y, y\right\rangle \\
& -(1-\lambda)\left\langle\left|\left(B^{*} B\right)^{\frac{1}{1-\lambda}}-\left\langle\left(B^{*} B\right)^{\frac{1}{1-\lambda}} y, y\right\rangle\right|^{r} y, y\right\rangle
\end{aligned}
$$

for any $x, y \in \mathcal{H}$, with $\|x\|=\|y\|=1$.

Theorem 3.1. Let $A, B \in B(\mathcal{H}), 0<\lambda<1$ and $r \geq 2$. Then

(3.3) $\left\|B^{*} A\right\|^{2 r} \leq \lambda\left\|\left(A^{*} A\right)^{\frac{r}{\lambda}}\right\|+(1-\lambda)\left\|\left(B^{*} B\right)^{\frac{r}{1-\lambda}}\right\|-\inf _{\|x\|=1} \zeta(x)-\inf _{\|y\|=1} \zeta(y)$,

where

$$
\begin{aligned}
& \zeta(x)=\lambda\left\langle\left|\left(A^{*} A\right)^{\frac{1}{\lambda}}-\left\langle\left(A^{*} A\right)^{\frac{1}{\lambda}} x, x\right\rangle\right|^{r} x, x\right\rangle, \\
& \zeta(y)=(1-\lambda)\left\langle\left|\left(B^{*} B\right)^{\frac{1}{1-\lambda}}-\left\langle\left(B^{*} B\right)^{\frac{1}{1-\lambda}} y, y\right\rangle\right|^{r} y, y\right\rangle .
\end{aligned}
$$

In addition,

$$
w^{2 r}\left(B^{*} A\right) \leq\left\|\lambda\left(A^{*} A\right)^{\frac{r}{\lambda}}+(1-\lambda)\left(B^{*} B\right)^{\frac{r}{1-\lambda}}\right\|-\inf _{\|x\|=1} \gamma(x)
$$

where

$\gamma(x)=\left\langle\left(\lambda\left|\left(A^{*} A\right)^{\frac{1}{\lambda}}-\left\langle\left(A^{*} A\right)^{\frac{1}{\lambda}} x, x\right\rangle\right|^{r}+(1-\lambda)\left|\left(B^{*} B\right)^{\frac{1}{1-\lambda}}-\left\langle\left(B^{*} B\right)^{\frac{1}{1-\lambda}} x, x\right\rangle\right|^{r}\right) x, x\right\rangle$. 
Proof. By taking supremum over $x, y \in \mathcal{H}$ with $\|x\|=\|y\|=1$ in inequality (3.2), we get the required inequality (3.3).

Putting $x=y$ in inequality (3.2), we obtain the numerical radius inequality (3.4).

Corollary 3.1. For $A, B \in B(\mathcal{H}), 0<\lambda<1$ and $r \geq 2$, the following inequalities hold:

$$
\begin{aligned}
|\langle A x, y\rangle|^{2 r} \leq & \lambda\left\langle\left(A^{*} A\right)^{\frac{r}{\lambda}} x, x\right\rangle-\lambda\left\langle\left|\left(A^{*} A\right)^{\frac{1}{\lambda}}-\left\langle\left(A^{*} A\right)^{\frac{1}{\lambda}} x, x\right\rangle\right|^{r} x, x\right\rangle+(1-\lambda), \\
\left|\left\langle A^{2} x, y\right\rangle\right|^{2 r} \leq & \lambda\left\langle\left(A^{*} A\right)^{\frac{r}{\lambda}} x, x\right\rangle-\lambda\left\langle\left|\left(A^{*} A\right)^{\frac{1}{\lambda}}-\left\langle\left(A^{*} A\right)^{\frac{1}{\lambda}} x, x\right\rangle\right|^{r} x, x\right\rangle \\
& +(1-\lambda)\left\langle\left(A A^{*}\right)^{\frac{r}{1-\lambda}} y, y\right\rangle-(1-\lambda)\left\langle\left|\left(A A^{*}\right)^{\frac{1}{1-\lambda}}-\left\langle\left(A A^{*}\right)^{\frac{1}{1-\lambda}} y, y\right\rangle\right|^{r} y, y\right\rangle,
\end{aligned}
$$

where $x, y \in \mathcal{H},\|x\|=\|y\|=1$.

Corollary 3.2. For $A, B \in B(\mathcal{H}), 0<\lambda<1$ and $r \geq 2$, the following norm inequalities and numerical radius inequalities hold:

(i) $\|A\|^{2 r} \leq \lambda\left\|\left(A^{*} A\right)^{\frac{r}{\lambda}}\right\|+(1-\lambda)-\inf _{\|x\|=1} \zeta(x)$;

(ii) $\left\|A^{2}\right\|^{2 r} \leq \lambda\left\|\left(A^{*} A\right)^{\frac{r}{\lambda}}\right\|+(1-\lambda)\left\|\left(A A^{*}\right)^{\frac{r}{1-\lambda}}\right\|-\inf _{\|x\|=1} \zeta(x)-\inf _{\|y\|=1} \zeta(y)$;

(iii) $w^{2 r}(A) \leq\left\|\lambda\left(A^{*} A\right)^{\frac{r}{\lambda}}+(1-\lambda) I\right\|-\inf _{\|x\|=1} \zeta(x)$, where

$$
\begin{aligned}
& \zeta(x)=\lambda\left\langle\left|\left(A^{*} A\right)^{\frac{1}{\lambda}}-\left\langle\left(A^{*} A\right)^{\frac{1}{\lambda}} x, x\right\rangle\right|^{r} x, x\right\rangle, \\
& \zeta(y)=(1-\lambda)\left\langle\left|\left(A A^{*}\right)^{\frac{1}{1-\lambda}}-\left\langle\left(A A^{*}\right)^{\frac{1}{1-\lambda}} y, y\right\rangle\right|^{r} y, y\right\rangle ;
\end{aligned}
$$

(iv) $w^{2 r}\left(A^{2}\right) \leq\left\|\lambda\left(A^{*} A\right)^{\frac{r}{\lambda}}+(1-\lambda)\left(A A^{*}\right)^{\frac{r}{1-\lambda}}\right\|-\inf _{\|x\|=1} \zeta(x)$, where

$$
\zeta(x)=\left\langle\left(\lambda\left|\left(A^{*} A\right)^{\frac{1}{\lambda}}-\left\langle\left(A^{*} A\right)^{\frac{1}{\lambda}} x, x\right\rangle\right|^{r}+(1-\lambda)\left|\left(A A^{*}\right)^{\frac{1}{1-\lambda}}-\left\langle\left(A A^{*}\right)^{\frac{1}{1-\lambda}} x, x\right\rangle\right|^{r}\right) x, x\right\rangle .
$$

We are going to establish a refinement of a numerical inequality for Hilbert space operators. We need the following lemmas. The first lemma is a generalization of the mixed Schwarz inequality.

Lemma 3.2. ([17, Lemma 2.1]). Let $A \in B(\mathcal{H})$ and $f$ and $g$ be nonnegative functions on $[0, \infty)$ which are continuous and satisfy the relation $f(t) g(t)=t$ for all $t \in[0, \infty)$. Then

$$
|\langle A x, y\rangle| \leq\|f(|A|) x\|\left\|g\left(\left|A^{*}\right|\right) y\right\|
$$

for all $x, y \in H$.

The next lemma is a consequence of the convexity of the function $f(t)=t^{r}, r \geq 1$.

Lemma 3.3. ([17, Lemma 2.3]). Let $a_{i}, i=1,2, \ldots, n$, be positive real numbers. Then

$$
\left(\sum_{i=1}^{n} a_{i}\right)^{r} \leq n^{r-1} \sum_{i=1}^{n} a_{i}^{r}, \quad \text { for } r \geq 1
$$


The following theorem is a generalization of the inequalities (1.3) and (1.4).

Theorem 3.2. ([17, Lemma 2.5]). Let $A_{i}, X_{i}, B_{i} \in B(\mathcal{H}), i=1,2, \ldots, n$, and let $f$ and $g$ be nonnegative functions on $[0, \infty)$ which are continuous and satisfy the relation $f(t) g(t)=t$ for all $t \in[0, \infty)$. Then

$$
w^{r}\left(\sum_{i=1}^{n} A_{i}^{*} X_{i} B_{i}\right) \leq \frac{n^{r-1}}{2}\left\|\sum_{i=1}^{n}\left(\left(B_{i}^{*} f^{2}\left(\left|X_{i}\right|\right) B_{i}\right)^{r}+\left(A_{i}^{*} g^{2}\left(\left|X_{i}^{*}\right|\right) A_{i}\right)^{r}\right)\right\|, \quad r \geq 1 .
$$

We refine the above inequality for $r \geq 1$ by applying a refinement of the HölderMcCarthy inequality. To achieve our next result, we utilize the strategy of [17, Lemma 2.5].

Theorem 3.3. Let $A_{i}, X_{i}, B_{i} \in B(\mathcal{H}), i=1,2, \ldots, n$, be invertible operators and let $f$ and $g$ be nonnegative functions on $[0, \infty)$ which are continuous and satisfy in $f(t) g(t)=t$ for all $t \in[0, \infty)$. Then, for all $r>1$,

$$
w^{r}\left(\sum_{i=1}^{n} A_{i}^{*} X_{i} B_{i}\right) \leq \frac{n^{r-1}}{2 \mu}\left\|\sum_{i=1}^{n}\left(B_{i}^{*} f^{2}\left(\left|X_{i}\right|\right) B_{i}\right)^{r}+\left(A_{i}^{*} g^{2}\left(\left|X_{i}^{*}\right|\right) A_{i}\right)^{r}\right\|,
$$

where $\mu=\min \{\zeta, \gamma\}, \zeta=\inf \left\{1+2(r-1)\left(1-\frac{\left\langle\left(B_{i}^{*} f^{2}\left(\left|X_{i}\right|\right) B_{i}\right)^{\frac{1}{2}} x, x\right\rangle}{\left\langle\left(B_{i}^{*} f^{2}\left(\left|X_{i}\right|\right) B_{i}\right) x, x\right\rangle^{\frac{1}{2}}}\right):\|x\|=1\right\}$ and $\gamma=\inf \left\{1+2(r-1)\left(1-\frac{\left\langle\left(A_{i}^{*} g^{2}\left(\left|X_{i}^{*}\right|\right) A_{i}\right)^{\frac{1}{2}} x, x\right\rangle}{\left\langle\left(A_{i}^{*} g^{2}\left(\left|X_{i}^{*}\right|\right) A_{i}\right) x, x\right\rangle^{\frac{1}{2}}}\right):\|x\|=1\right\}$.

Proof. For every unit vector $x \in H$, we have

$$
\begin{aligned}
& \left|\left\langle\left(\sum_{i=1}^{n} A_{i}^{*} X_{i} B_{i}\right) x, x\right\rangle\right|^{r}=\left|\sum_{i=1}^{n}\left\langle\left(A_{i}^{*} X_{i} B_{i}\right) x, x\right\rangle\right|^{r} \\
\leq & \left(\sum_{i=1}^{n}\left|\left\langle A_{i}^{*} X_{i} B_{i} x, x\right\rangle\right|\right)^{r}=\left(\sum_{i=1}^{n} \mid\left\langle X_{i} B_{i} x, A_{i} x\right\rangle\right)^{r} \\
\leq & \left(\sum_{i=1}^{n}\left\langle f^{2}\left(\left|X_{i}\right|\right) B_{i} x, B_{i} x\right\rangle^{\frac{1}{2}}\left\langle g^{2}\left(\left|X_{i}^{*}\right|\right) A_{i} x, A_{i} x\right\rangle^{\frac{1}{2}}\right)^{r}
\end{aligned}
$$

(by Lemma 3.2)

$$
\leq n^{r-1} \sum_{i=1}^{n}\left\langle B_{i}^{*} f^{2}\left(\left|X_{i}\right|\right) B_{i} x, x\right\rangle^{\frac{r}{2}}\left\langle A_{i}^{*} g^{2}\left(\left|X_{i}^{*}\right|\right) A_{i} x, x\right\rangle^{\frac{r}{2}}
$$

(by Lemma 3.3)

$$
\begin{aligned}
= & n^{r-1} \sum_{i=1}^{n}\left(\left\langle B_{i}^{*} f^{2}\left(\left|X_{i}\right|\right) B_{i} x, x\right\rangle^{r}\right)^{\frac{1}{2}}\left(\left\langle A_{i}^{*} g^{2}\left(\left|X_{i}^{*}\right|\right) A_{i} x, x\right\rangle^{r}\right)^{\frac{1}{2}} \\
\leq & \frac{n^{r-1}}{2}\left(\sum_{i=1}^{n}\left(\left\langle B_{i}^{*} f^{2}\left(\left|X_{i}\right|\right) B_{i} x, x\right\rangle^{r}+\left\langle A_{i}^{*} g^{2}\left(\left|X_{i}^{*}\right|\right) A_{i} x, x\right\rangle^{r}\right)\right) \\
& \quad(\text { by } \mathrm{AM}-\mathrm{GM})
\end{aligned}
$$




$$
\begin{aligned}
& \leq \frac{n^{r-1}}{2}\left(\sum _ { i = 1 } ^ { n } \left(\frac{1}{\zeta(x)}\left\langle\left(B_{i}^{*} f^{2}\left(\left|X_{i}\right|\right) B_{i}\right)^{r} x, x\right\rangle+\frac{1}{\gamma(x)}\left\langle\left( A_{i}^{*} g^{2}\left(\mid X_{i}^{*}\right)\right.\right.\right.\right. \\
& \quad \quad \frac{n^{r-1}}{2 \mu} \sum_{i=1}^{n}\left\langle\left(\left(B_{i}^{*} f^{2}\left(\left|X_{i}\right|\right) B_{i}\right)^{r}+\left(A_{i}^{*} g^{2}\left(\left|X_{i}^{*}\right|\right) A_{i}\right)^{r}\right) x, x\right\rangle \\
& =\frac{n^{r-1}}{2 \mu}\left\langle\sum_{i=1}^{n}\left(\left(B_{i}^{*} f^{2}\left(\left|X_{i}\right|\right) B_{i}\right)^{r}+\left(A_{i}^{*} g^{2}\left(\left|X_{i}^{*}\right|\right) A_{i}\right)^{r}\right) x, x\right\rangle .
\end{aligned}
$$

Therefore, by taking supremum over $x \in \mathcal{H}$ with $\|x\|=1$, we have the desired relation.

If we assume that $f(t)=t^{\lambda}$ and $g(t)=t^{1-\lambda}, 0<\lambda<1$, in Theorem 3.3, then we get the following corollary.

Corollary 3.3. Let $A_{i}, X_{i}, B_{i} \in B(\mathcal{H}), i=1,2, \ldots, n$, be invertible operators, $r>1$ and $0<\lambda<1$. Then

$$
w^{r}\left(\sum_{i=1}^{n} A_{i}^{*} X_{i} B_{i}\right) \leq \frac{n^{r-1}}{2 \mu}\left\|\sum_{i=1}^{n}\left(B_{i}^{*}\left|X_{i}\right|^{2 \lambda} B_{i}\right)^{r}+\left(A_{i}^{*}\left|X_{i}^{*}\right|^{2(1-\lambda)} A_{i}\right)^{r}\right\|,
$$

where $\mu=\min \{\zeta, \gamma\}$,

$$
\begin{aligned}
& \zeta=\inf \left\{1+2(r-1)\left(1-\frac{\left\langle\left(B_{i}^{*}\left|X_{i}\right|^{2 \lambda} B_{i}\right)^{\frac{1}{2}} x, x\right\rangle}{\left\langle\left(B_{i}^{*}\left|X_{i}\right|^{2 \lambda} B_{i}\right) x: x\right\rangle^{\frac{1}{2}}}\right):\|x\|=1\right\}, \\
& \gamma=\inf \left\{1+2(r-1)\left(1-\frac{\left\langle\left(A_{i}^{*}\left|X_{i}\right|^{2(1-\lambda)} A_{i}\right)^{\frac{1}{2}} x, x\right\rangle}{\left\langle\left(A_{i}^{*}\left|X_{i}\right|^{2(1-\lambda)} A_{i}\right) x, x\right\rangle^{\frac{1}{2}}}\right):\|x\|=1\right\} .
\end{aligned}
$$

In particular,

$$
w\left(\sum_{i=1}^{n} A_{i}^{*} X_{i} B_{i}\right) \leq \frac{1}{2}\left\|\sum_{i=1}^{n}\left(B_{i}^{*}\left|X_{i}\right| B_{i}+A_{i}^{*}\left|X_{i}^{*}\right| A_{i}\right)\right\| .
$$

Setting $A_{i}=B_{i}=I, i=1,2, \cdots, n$, in Theorem 3.3, the following inequalities for sums of operators are obtained.

Corollary 3.4. Let $X_{i} \in B(\mathcal{H}), i=1,2, \ldots, n$, be invertible operators and $f$ and $g$ be continuous nonnegative functions on $[0, \infty)$, such that $f(t) g(t)=t$ for all $t \in[0, \infty)$. Then, for $r>1$,

$$
w^{r}\left(\sum_{i=1}^{n} X_{i}\right) \leq \frac{n^{r-1}}{2 \mu}\left\|\sum_{i=1}^{n}\left(f^{2 r}\left(\left|X_{i}\right|\right)+g^{2 r}\left(\left|X_{i}^{*}\right|\right)\right)\right\|
$$


where $\mu=\min \{\zeta, \gamma\}$,

$$
\begin{aligned}
& \zeta=\inf \left\{1+2(r-1)\left(1-\frac{\left\langle f\left(\left|X_{i}\right|\right) x, x\right\rangle}{\left\langle f^{2}\left(\left|X_{i}\right|\right) x, x\right\rangle^{\frac{1}{2}}}\right):\|x\|=1\right\}, \\
& \gamma=\inf \left\{1+2(r-1)\left(1-\frac{\left\langle g\left(\left|X_{i}^{*}\right|\right) x, x\right\rangle}{\left\langle g^{2}\left(\left|X_{i}^{*}\right|\right) x, x\right\rangle^{\frac{1}{2}}}\right):\|x\|=1\right\} .
\end{aligned}
$$

In particular,

$$
w^{r}\left(\sum_{i=1}^{n} X_{i}\right) \leq \frac{n^{r-1}}{2 \mu}\left\|\sum_{i=1}^{n}\left|X_{i}\right|^{2 \lambda r}+\left|X_{i}^{*}\right|^{2(1-\lambda) r}\right\|, \quad \lambda \in(0,1),
$$

where $\mu=\min \{\zeta, \gamma\}$,

$$
\begin{aligned}
& \zeta=\inf \left\{1+2(r-1)\left(1-\frac{\left\langle\left|X_{i}\right|^{\lambda} x, x\right\rangle}{\left\langle\left|X_{i}\right|^{2 \lambda} x, x\right\rangle^{\frac{1}{2}}}\right):\|x\|=1\right\}, \\
& \gamma=\inf \left\{1+2(r-1)\left(1-\frac{\left\langle\left|X_{i}^{*}\right|^{(1-\lambda)} x, x\right\rangle}{\left\langle\left|X_{i}^{*}\right|^{2(1-\lambda)} x, x\right\rangle^{\frac{1}{2}}}\right):\|x\|=1\right\} .
\end{aligned}
$$

If $\lambda=\frac{1}{2}$ in above inequality, we get

$$
w^{r}\left(\sum_{i=1}^{n} X_{i}\right) \leq \frac{n^{r-1}}{2 \mu}\left\|\sum_{i=1}^{n}\left|X_{i}\right|^{r}+\left|X_{i}^{*}\right|^{r}\right\|, \quad r \geq 1,
$$

where $\mu=\min \{\zeta, \gamma\}$,

$$
\begin{aligned}
& \zeta=\inf \left\{1+2(r-1)\left(1-\frac{\left\langle\left|X_{i}\right|^{\frac{1}{2}} x, x\right\rangle}{\left\langle\left|X_{i}\right| x, x\right\rangle^{\frac{1}{2}}}\right):\|x\|=1\right\}, \\
& \gamma=\inf \left\{1+2(r-1)\left(1-\frac{\left\langle\left|X_{i}^{*}\right|^{\frac{1}{2}} x, x\right\rangle}{\left\langle\left|X_{i}^{*}\right| x, x\right\rangle^{\frac{1}{2}}}\right):\|x\|=1\right\} .
\end{aligned}
$$

Letting $n=1$ in inequality (3.3), we obtain

$$
w^{r}(X) \leq \frac{1}{2 \mu}\left\||X|^{r}+\left|X^{*}\right|^{r}\right\|,
$$

where $\mu=\min \{\zeta, \gamma\}$,

$$
\begin{aligned}
& \zeta=\inf \left\{1+2(r-1)\left(1-\frac{\left\langle|X|^{\frac{1}{2}} x, x\right\rangle}{\langle|X| x, x\rangle^{\frac{1}{2}}}\right):\|x\|=1\right\}, \\
& \gamma=\inf \left\{1+2(r-1)\left(1-\frac{\left\langle\left|X^{*}\right|^{\frac{1}{2}} x, x\right\rangle}{\left\langle\left|X^{*}\right| x, x\right\rangle^{\frac{1}{2}}}\right):\|x\|=1\right\} .
\end{aligned}
$$

Next, we present some numerical radius inequalities for products of operators. Put $X_{i}=I, i=1,2, \ldots, n$, in Theorem 3.3, to get the following. 
Corollary 3.5. Let $A_{i}, B_{i} \in B(\mathcal{H}), i=1,2, \ldots, n$, be invertible operators and $r \geq 1$. Then

$$
w^{r}\left(\sum_{i=1}^{n} A_{i}^{*} B_{i}\right) \leq \frac{n^{r-1}}{2 \mu}\left\|\sum_{i=1}^{n}\left|B_{i}\right|^{2 r}+\left|A_{i}\right|^{2 r}\right\|,
$$

where $\mu=\min \{\zeta, \gamma\}$,

$$
\begin{aligned}
& \zeta=\inf \left\{1+2(r-1)\left(1-\frac{\left\langle\left|B_{i}\right| x, x\right\rangle}{\left\langle\left|B_{i}\right| x, x\right\rangle^{\frac{1}{2}}}\right):\|x\|=1\right\}, \\
& \gamma=\inf \left\{1+2(r-1)\left(1-\frac{\left\langle\left|A_{i}\right| x, x\right\rangle}{\left\langle\left|A_{i}\right| x, x\right\rangle^{\frac{1}{2}}}\right):\|x\|=1\right\} .
\end{aligned}
$$

In particular,

$$
w\left(\sum_{i=1}^{n} A_{i}^{*} B_{i}\right) \leq \frac{1}{2}\left\|\sum_{i=1}^{n}\left(B_{i}^{*} B_{i}+A_{i}^{*} A_{i}\right)\right\| .
$$

Remark 3.1. If we set $n=1$ in Corollary 3.5, then

$$
w^{r}\left(A^{*} B\right) \leq \frac{1}{2 \mu}\left\|\left(B^{*} B\right)^{r}+\left(A^{*} A\right)^{r}\right\|,
$$

where $\mu=\min \{\zeta, \gamma\}$,

$$
\begin{aligned}
& \zeta=\inf \left\{1+2(r-1)\left(1-\frac{\left\langle\left(B^{*} B\right)^{\frac{1}{2}} x, x\right\rangle}{\left\langle\left(B^{*} B\right) x, x\right\rangle^{\frac{1}{2}}}\right):\|x\|=1\right\}, \\
& \gamma=\inf \left\{1+2(r-1)\left(1-\frac{\left\langle\left(A^{*} A\right)^{\frac{1}{2}} x, x\right\rangle}{\left\langle\left(A^{*} A\right) x, x\right\rangle^{\frac{1}{2}}}\right):\|x\|=1\right\} .
\end{aligned}
$$

Acknowledgements. The authors would like to thank the referees for several useful comments.

\section{REFERENCES}

[1] S. S. Dragomir, Vector inequalities for powers of some operators in Hilbert spaces, Filomat 23(1) (2009), 69-83.

[2] S. S. Dragomir, A note on new refinements and reverses of Young's inequality, Transylvanian Journal of Mathematics and Mechanics 8(1)(2016), 46-49.

[3] M. Fujii and R. Nakamoto, Refinements of Hölder-McCarthy inequality and Young inequality, Adv. Oper. Theory 1(2) (2016), 184-188.

[4] K. E. Gustafson and D. K. M. Rao, Numerical Range, Springer-Verlag, New York, 1997.

[5] J. A. R. Holbrook, Multiplicative properties of the numerical radius in operator theory, J. Reine Angew. Math. 237 (1969), 166-174.

[6] M. Kian, Operator Jensen inequality for superquadratic functions, Linear Algebra Appl. 456 (2014), 82-87.

[7] F. Kittaneh, A numerical radius inequality and an estimate for the numerical radius of the Frobenius companion matrix, Studia Math. 158(1) (2003), 11-17. 
[8] F. Kittaneh, Numerical radius inequalities for Hilbert space operators, Studia Math. 168(1) (2005), 73-80.

[9] F. Kittaneh and M. El-Haddad, Numerical radius inequalities for Hilbert space operators II, Studia Math. 182(2) (2007), 133-140.

[10] F. Kittaneh, M. S. Moslehian and T. Yamazaki, Cartesian decomposition and numerical radius inequalities, Linear Algebra Appl. 471 (2015), 46-53.

[11] H. Kober, On the arithmetic and geometric means and on Hölder's inequality, Proc. Amer. Math. Soc. 9 (1958), 452-459.

[12] C-S. Lin and Y. J. Cho, On Hölder-McCarthy-type inequalities with powers, J. Korean Math. Soc. 39(3) (2002), 351-361.

[13] M. Sattari, M. S. Moslehian and T. Yamazaki, Some generalized numerical radius inequalities for Hilbert space operators, Linear Algebra Appl. 470 (2015), 216-227.

[14] M. Sababheh, Heinz-type numerical radii inequalities, Linear Multilinear Algebra, DOI 10.1080/03081087.2018.1440518.

[15] M. Sababheh, Numerical radius inequalities via convexity, Linear Algebra Appl. 549 (2018), 67-78.

[16] Y. Seo, Hölder type inequalities on Hilbert $C^{*}$-modules and its reverses, Ann. Funct. Anal. 5(1) (2014), 1-9.

[17] K. Shebrawi and H. Albadwi, Numerical radius and operator norm inequalities, J. Inequal. Appl. (2009), Article ID 492154, 11 pages.

[18] K. Shebrawi, Numerical radius inequalities for certain $2 \times 2$ operator matrices II, Linear Algebra Appl. 523 (2017), 1-12.

[19] A. Zamani, Some lower bounds for the numerical radius of Hilbert space operators, Adv. Oper. Theory 2(2) (2007), 98-107.

${ }^{1}$ Department of Mathematics, Mashhad Branch,

ISLAMIC AZAD UNIVERSITY, MASHHAD, IRAN

Email address: zheydarbeygi@yahoo.com

Email address: maryam_amyari@yahoo.com

Email address: amyari@mshdiau.ac.ir

*Corresponding AUthor 